\title{
Risikomanagement und Patientensicherheit in der Behandlung
}

\section{Ein Leitfaden: Prinzipien und praktische Ansätze}

Herausgeber und Verfasser: Richard Cranovsky und Hans Hurter Verlag: H+ Bildungszentrum, Aarau, August 2003

In übersichtlicher Art und Weise zeigen die Autoren den Status quo des Risikomanagements und der Patientensicherheit in der medizinischen Versorgung auf. Detailliert und anhand konkreter Beispiele werden Möglichkeiten der Risikominderung im klinischen Alltag diskutiert. Abgerundet wird der Leitfaden durch eine internationale Übersicht laufender Programme zur Erhöhung der Patientensicherheit und ein umfangreiches Literaturverzeichnis zur vertiefenden Lektüre.

Einleitend wird in übersichtlicher Art die verwendete Fachterminologie beschrieben und erklärt und das vieldiskutierte Ausmass des Problems unerwünschter Ereignisse und Fehler dargestellt. Nach einer Übersicht über mögliche Ursachen unerwünschter Ereignisse sowohl auf systemischer wie menschlicher Ebene werden Grundsätze und Implementierungsmöglichkeiten von Massnahmen zur Risikominderung vorgestellt. Anhand konkreter Fallstudien werden anschliessend spezifische Probleme einzelner Versorgungsbereiche diskutiert und konkrete Empfehlungen gegeben. Im letzten Teil des Buches werden internationale gesundheitspolitische Programme zu Patientensicherheit und
Risikomanagement vorgestellt. Der eine oder andere mag sich eine etwas eingehendere Analyse der Aspekte der Organisationsentwicklung und Systemanalyse wünschen; er sei auf die umfangreiche Bibliographie mit zusätzlichen Internet-Links $\mathrm{zu}$ wichtigen und aktuellen Quellen verwiesen.

\section{Fazit}

Der Leitfaden ermöglicht einen raschen und abwechslungsreichen Einstieg in die Thematik des Risikomanagements und gibt einen guten Überblick zum aktuellen Stand des Wissens.

Dr. med. Georg von Below, Bern 